\title{
Los proyectos de caminata rumbo a la escuela para el conocimiento del entorno y favorecer actitudes y hábitos saludables desde educación infantil
}

\author{
ROBERTO SILVA PIŃEIRO* \\ Universidade de Vigo - España \\ Recibido el 28-05-2017; primera evaluación el 18-06-2018; \\ segunda evaluación el 12-07-2018; aceptado el 14-07-2018
}

\section{RESUMEN}

En la actualidad, una mayoría de niños no acude solo a escuela, lo que implica que no adquiera algunas competencias que favorezcan la autonomía y la interacción social. Las autoridades mundiales animan a promover entornos seguros y saludables próximos al colegio, pues, desde hace décadas, tienen resultados positivos en varios países. Los centros escolares espańoles de educación infantil y primaria están reconocidos como lugares apropiados para fomentar la experimentación y conocimiento del entorno a través de la actividad física y el juego. Entre los proyectos de entorno escolar activo, destacan los relativos al camino escolar, normalmente impulsados por los gobiernos locales, con la estrecha colaboración del colegio. En cualquier propuesta similar, es importante prestar atención tanto a la comunidad educativa como a los espacios urbanos.

El trayecto a la escuela, que se realiza al menos dos veces a día, se puede efectuar andando o en bicicleta, lo que puede ser una alternativa fabulosa y modificable para aumentar la actividad física diaria de nińos y adolescentes, pues ayudan a los nińos y jóvenes a mejorar su salud física (fuerza, resistencia aeróbica, equilibrio, coordinación y agilidad), relaciones sociales y habilidades personales.

La idea del proyecto caminando a la escuela es una iniciativa que intenta educar a la sociedad en los desplazamientos activos y urbanismo sostenible, con

\footnotetext{
* Profesor Departamento Didácticas Especiales. Facultad de Ciencias de la Educación y el Deporte. Campus A Xunqueira. Pontevedra (España). Licenciado en Ciencias de la Actividad Física y el Deporte. Universidad A Coruña. Diplomado en Educación Física. Universidad Camilo José Cela Máster en Dirección de Instalaciones Deportivas. Universidad San Jorge. Gerente del Colegio Profesional de la Educación Física y el Deporte de Galicia. Miembro del Grupo de Investigación REMOSS. Universidad de Vigo. Correo: roberto.silva@uvigo.es
} 
ejemplos por todo el mundo. En cualquier caso, el protagonista del camino escolar es el alumno, que aprenderá a desplazarse con seguridad y por sus propios medios, y a reflexionar en actividades de aula sobre movilidad y mejora ambiental, promocionadas por directivos y profesores.

Palabras-clave: entorno escolar, caminata, hábitos saludables.

\section{The projects of walk towards the school for the knowledge of the environment and to favor the healthy attitudes and habits from childhood education}

\section{Abstract}

Currently a majority of children do not go to school alone, which means that they do not reach some skills that favor autonomy and social interaction. The world authorities are motivating safe and healthy environments around the school, because for decades they have had positive results in several countries. Spanish kindergardens and primary education schools are recognized as appropriate places to experience and knowledge of the environment through physical activity and play. Among the projects on an active school environment, those related with the road to the school are relevant, normally promoted by local governments, with the close collaboration of the school. In any similar proposal, it is as important to pay attention to the educational community like as urban spaces.

The walk to the school, which takes place at least twice a day, can be done on foot or by bicycle, which can be a fabulous and modifiable alternative to increase the daily physical activity of children and adolescents, because it helps them to improve their physical health (strength, aerobic endurance, balance, coordination and agility), social relationships and personal skills.

The idea of the project walking to school is an initiative that tries to educate to the society into active displacements and sustainable urbanism, with examples all over the world. In any case, the protagonist of the walk to the school is the student, who will learn to move safely and by their own means, and to reflect on classroom activities on mobility and environmental improvement.

Keywords: school environment, walks, healthy habits

\section{Os projetos de caminhar em direçáo à escola para o conhecimento do} ambiente e favorecer as atitudes e hábitos saudáveis desde a educaçáo infantil

\section{RESUMO}

Atualmente, a maioria das crianças viaxan acompanhadas até a escola, o que significa que elas não adquirem algumas habilidades que favorecem a autonomia e a interação social. As autoridades globais encorajam a promoção de ambientes seguros e saudáveis em toda a escola, porque durante décadas foram alcançados resultados positivos em vários países. As escolas infantis e primárias da Espanha são 
reconhecidas como lugares adequados para experimentar e conhecer o ambiente através da atividade física e do jogo. Os projetos de escolas ativas incluem aqueles relacionados co caminho para a escola, geralmente conduzidos por governos locais, com a estreita colaboração da própria escola. Para qualquer proposta similar, é importante prestar atençáo à comunidade educativa e também aos espaços abertos

A ida à escola, que acontece pelo menos duas vezes ao dia, pode ser feita a pé ou de bicicleta, o que pode ser uma alternativa fabulosa e modificável para aumentar a atividade física diária de crianças e adolescentes, ajudando-os a melhorar sua saúde. físico (força, resistência aeróbica, equilíbrio, coordenação e agilidade), relaçóes sociais e habilidades pessoais.

A ideia do projeto caminhando para a escola é uma iniciativa que tenta educar a sociedade em deslocamentos ativos e urbanismo sustentável, com exemplos em todo o mundo. Em qualquer caso, o protagonista do caminho para a escola é o aluno, que aprenderá a se locomover com segurança e por seus próprios meios, e a refletir nas atividades de aula sobre mobilidade e melhoria ambiental.

Palabras-chave: ambiente escolar, caminhada, hábitos saudaveis.

\section{INTRODUCCIÓN}

Entre los importantes cambios sociales que se han ido produciendo en las últimas décadas, nos encontramos que las familias comienzan a delegar sus funciones a la escuela, que cada vez más se contempla como un destacado lugar de socialización y promoción de hábitos para la vida. Así pues, la escuela y su entorno, se consideran elementos básicos del contexto vital de aprendizaje, durante el horario lectivo y más allá del mismo.

No es lo habitual, pero desde el centro educativo se podría contribuir a fomentar el hábito de desplazamiento activo a la escuela, y favorecer su evaluación, apoyándose por ejemplo en la educación física (Villa y Pérez, 2014). La escuela siempre ha sido un lugar idóneo para fomentar hábitos activos, pues de forma económica se puede acceder a gran cantidad de población en edad escolar, y durante esta etapa es mayor la predisposición a adquirir hábitos saludables.

Aunque se presentarán datos de varios países, la experiencia sobre la que vamos a trabajar tiene lugar en el contexto español.

\section{EL ENTORNO ESCOLAR COMO ESPACIO SEGURO Y SALUDABLE}

El Fondo de las Naciones Unidas para la Infancia alude a la responsabilidad ética de garantizar que las escuelas puedan proveer un ambiente seguro, pues este hecho promueve el aprendizaje del estudiante y su crecimiento saludable 
(Devine y Cohen, 2007). Son muy pocos los que dudan de la necesidad de un ambiente sano y solidario con el medio (Gavidia, 2001; Menéndez y Moreno, 2006), donde los nińos puedan desarrollarse plenamente con el mayor grado de bienestar posible (Confederación Española de Asociaciones de Padres de Alumnos, 2012; Hayes, 2010).

Cada vez más evidente, entre la ciudadanía, existe una sensación subjetiva de inseguridad que implica una reducción de actividades. La construcción de aceras y carriles para peatones y ciclistas reduce esta percepción; a la vez que los caminos escolares y el aumento de actividad física disminuyen los costes del bus escolar, bajas laborales, enfermedades, contaminación de vehículos a motor y aparcamiento (Sælensminde, 2004). El fomento de entornos educativos seguros y saludables es un objetivo compartido por organizaciones y profesionales de la educación, que tiene su reflejo en el ambiente institucional y físico de la escuela, así como en los comportamientos que se intentan promover (Meresman, 2005). En este sentido, la European Heart Network (EHN) (2001) anima a los centros educativos y gobiernos locales a promover la actividad física para la salud, estableciendo espacios y rutas seguras en torno a las escuelas para los desplazamientos a pie y en bicicleta.

Que un niño se desplace de forma activa al colegio, caminando o en bicicleta, es una oportunidad para mejorar la condición física y, al ser un comportamiento que se realiza diariamente como mínimo dos veces al día, es modificable, lo que podría garantizar niveles adecuados de salud en la población joven (Villa y Pérez, 2014). Existe evidencia científica de que los jóvenes que se desplazan al colegio en bicicleta tienen mayores niveles de condición física, en comparación con los que hacen de forma pasiva (Chillón et al., 2012), así como menores niveles de grasa (Lubans, Boreham, Kelly y Foster, 2011).

\section{MoVILIDAD ACTIVA, NiŃOS AUTÓNOMOS}

Entre 1970-1990, el número de niños europeos de 7-8 años que acudían solos al colegio se redujo al 9\%, y hoy en día los padres normalmente acompañan a sus hijos (Laria del Vals et al., 2014). La situación es similar en España, puesto que, en las ciudades, el $70 \%$ de los alumnos de educación primaria no van solos, y de los que acuden solos, la edad media supera los 9 años (Alonso, Esteban, Calatayud y Alamar, 2009). En 1970 la totalidad de los niños iban solos; en 1990, solo la mitad; en 2000, el 25\% (Román y Salís, 2010), y en la actualidad este porcentaje es todavía menor.

Acompañar, fundamentalmente, en coche y de forma sistemática a los niños al centro escolar cuando ya han crecido suficiente, acarrea repercusiones 
sobre su desarrollo psicomotor y social, pues se entorpece el aprendizaje autónomo y se disminuye el ejercicio físico diario y la interacción con las personas y el entorno durante el camino. En un análisis sobre los caminos escolares de 124 escuelas de Nueva York, se estimó que el beneficio a largo plazo sobre la reducción de las lesiones y ahorro socio-sanitario derivado de la accidentalidad en la movilidad, era 23 veces mayor que el coste (Muennin, Epstein, Li y DiMaggio, 2014).

Considerando los cambios hacia hábitos de vida que puede generar un entorno escolar, sin duda que la actividad física es uno de los factores más destacados para mantener y mejorar la salud. La actividad física regular ayuda a prevenir varias enfermedades crónicas (Warburton, Nicol y Bredin, 2006), mejorando la calidad de vida. La Organización Mundial de la Salud (WHO, por sus siglas en inglés) recomienda, entre los 5-17 años, realizar al menos 60 minutos diarios de actividad física entre moderada y vigorosa (WHO, 2010), pese a que la Comisión Europea (EC, por sus siglas en inglés) (2014) asegura que el $44 \%$ de los adultos europeos no alcance a realizar 150 minutos semanales.

El desplazamiento activo al colegio, andando o en bicicleta, puede ser una alternativa muy positiva para incrementar la actividad física diaria de nińos y adolescentes, pues ayuda a reducir el peso corporal (Sirard, Ainsworth, Mclver y Pate, 2005) y los factores de riesgo cardiovascular en los futuros adultos (Hamer y Chida, 2008). A pesar de ello, el desplazamiento activo al colegio ha ido cayendo en los últimos 30 ańos (Westerterp y Speakman, 2008), por diversos motivos (Davison, Werder y Lawson, 2008).

Entre ellos, encontramos la situación familiar, y así, en un trabajo desarrollado en 721 familias espańolas con niños y niñas de 6 a 12 años, cuando el padre y la madre estaban desempleados, el desplazamiento activo al colegio de sus hijos se incrementaba, al igual que cuando los padres se desplazaban activamente a su trabajo (Villa-González et al., 2012). Sin embargo, cuando el horario de padres e hijos coincidía, la probabilidad de que los niños acudiesen al colegio activamente disminuía (McMillan, 2007). Asimismo, aquellos que viven más cerca del colegio se desplazan más activamente que los que residen más alejados; y los alumnos de colegios públicos son más activos en sus desplazamientos que los de centros privados, pues normalmente estos están más alejados (Spallek, Turner, Spinks, Bain y McClure, 2006).

Según la Danish National Board of Health (DNBH), en Dinamarca el 60\% de nińos entre 11-15 ańos no alcanzaban los 60 minutos de actividad física diaria, y la proporción de los que no caminaban al colegio había aumentado un 200\% entre 1976-2006, por lo que su gobierno comenzó a introducir 
medidas estructurales para promocionar el transporte escolar activo a bajo coste (DNBH, 2006). Estudios publicados por la Danish Cancer Society (DCS) (2012) constataron que el $80 \%$ de sus municipios y $37 \%$ de sus escuelas pusieron en marcha iniciativas y aumentaron el número de niños que iban en bicicleta y a pie a la escuela al $80 \%$ y $65 \%$ respectivamente.

Por otra parte, desplazarse en bicicleta o a pie también genera empleo (WHO, 2014), pero sobre todo reduce el elevadísimo impacto que tiene el tráfico, tanto en el medio ambiente como en la salud de las personas. Para la Organización de Consumidores y Usuarios Españoles (OCU) (2012), la movilidad urbana activa ayuda a reducir las emisiones del transporte y mejorar el entorno, pero el tráfico de vehículos sigue siendo una de las principales causas de contaminación en los accesos a centros escolares, sobre todo durante la hora de llegada. Por otra parte, la Organización de Naciones Unidas (ONU) considera que las consecuencias de los accidentes de tráfico suponen un elevado coste público (Peden et al., 2004), y la protección de peatones y ciclistas ha pasado a ser prioridad internacional (ONU, 2009). Aunque los expertos afirman que se podrían prevenir hasta el 90\% de lesiones (MacKay y Vincenten, 2012), en Europa, los accidentes en la vía pública siguen siendo la principal causa de muerte entre los 5-19 ańos, probablemente debido a su mayor exposición e imprudencia de sus conductas (WHO, 2016), lo que coincide con lo que piensan también la Asociación Española de Pediatría de Atención Primaria (AEPAP) (2014).

\section{Algunas medidas de Seguridad para los proyectos de Caminata ACTIVA A LA ESCUELA}

En primer lugar, es importante que exista una comunicación e información fluida entre toda la comunidad educativa, que ayude a estar en contacto a los niños, familias, directivos, profesores y autoridades competentes (Xunta de Galicia, 2015). En los entornos urbanos o rurales tranquilos, no es necesario tomar una serie de medidas de control y seguridad, que en aquellos en el que el nivel de peligrosidad o desamparo del entorno del centro educativo sea alto.

Es necesario que se realicen varios debates abiertos y se elabore un documento operativo y de seguridad que conozcan sobre todo las familias, puesto que serán los beneficiarios de los aciertos o errores del mismo.

Entre las medidas de seguridad que se pueden poner en práctica en los proyectos de caminata activa a la escuela están: 
- Realizar un mapeo con señalética e indicación de los recursos disponibles que conozca toda la comunidad educativa.

- Solicitar y coordinar una serie de colaboradores entre los vecinos y comerciantes, que serán los encargados de informar a los niños, atender de su buen camino, y acogerlos en caso de incidencia. Estos colaboradores tendrán un distintivo visible en la puerta del establecimiento.

- Diseñar los itinerarios en función de la mayor seguridad posible, atendiendo tanto a la peligrosidad en los lugares de paso (tráfico, paso de peatones, semáforos...) como conflictividad social o criminal.

- Realizar los itinerarios en grupo, acompañados de adultos que vistan ropas brillantes.

- Proponer la realización de buses escolares a pie, con uno o varios padres que controlen y acompañen el itinerario, con paradas establecidas y controladas por adultos.

- Establecer puntos de control, en los que puedan encontrarse agentes de policía que controlen el tráfico y otras posibles incidencias.

- Identificar los lugares más peligrosos para buscar alternativas o aumentar el control sobre los mismos.

- Educar a los niños y los padres en el respecto a las normas de circulación y el civismo en los desplazamientos hacia la escuela, evitando que crucen por lugares inadecuados o sin respetar los semáforos o espacios de tránsito: caminar por la acera, y si no hay acera y debe caminar en la calle, camine de frente al tráfico; antes de cruzar la calle, deténgase y mire a la izquierda, derecha e izquierda nuevamente para ver si vienen automóviles; nunca salga precipitado frente a un auto estacionado; nunca camine mientras envíe mensajes de texto, habla por teléfono o use auriculares.

- Si su desplazamiento es en bicicleta, use siempre el casco, y asegúrese de que esté ajustado. Los niños necesitan saber las reglas de la carretera: mantenga una sola fila en el lado derecho de la carretera y pare completamente ante de cruzar la calle y camine con la bicicleta. Esté atento a la apertura de puertas de automóviles y use señales con la mano cuando vaya a realizar un cambio de dirección ((New Zealand Transport Agency, 2009; Román y Salís, 2010; Mackay y Vicenten, 2012; Silva y Daquinta, 2016).

Asimismo, la Organización Panamericana de la Salud (2011) establece otras medidas, entre las que destacan:

- Desarrollar dispositivos calmantes del tráfico como pasos altos y aceras, y así evitar también las invasiones de vía. 
- Aumentar las zonas peatonales y de tráfico «tranquilo» o reducido a determinadas horas.

- Repartir ropa reflectante entre los peatones, sobre todo en lugares de baja visibilidad y sin acera.

- Establecer límites de velocidad de 20, 30 y $50 \mathrm{~km} / \mathrm{h}$ según la peligrosidad de la vía

\section{ENFOQUE EDUCATIVO DEL ENTORNO Y VIDA ACTIVA EN EDUCACIÓN INFANTIL Y PRIMARIA}

En España, el Ministerio de Educación, a través del Real Decreto 1630/2006 contempla que el segundo ciclo de escolarización infantil se focalice, progresivamente, entre otros aspectos, al descubrimiento del entorno a través de la caminata, el movimiento, y las pautas elementales de convivencia y relación social. Cada centro escolar tiene una opción, una excelente oportunidad para completar los contenidos fijados por medio de actividades significativas, teniendo en cuenta que el entorno del alumnado se amplía y se diversifica, lo que les sitúa ante experiencias nuevas, de forma conveniente, mediante el juego.

El área curricular de «conocimiento de sí mismo y autonomía personal» plantea que los niños generen su identidad a través de las experiencias interactivas con el medio físico, natural y social. Serán fundamentales aquellas personas que les rodean, que les puedan ayudar y servir de ejemplo, así como la adquisición de hábitos saludables y de higiene, pues favorecen la autonomía personal y el respecto por los espacios vitales. De ahí que la escuela sea un lugar apropiado para fomentar, desde las primeras edades, los procesos exploratorios y de autoconocimiento. Al concluir esta etapa educativa, todo aquello que el niño haya vivenciado, fundamentalmente aquellas experiencias producidas a través de la actividad física en el entorno físico y natural, se convertirán en los cimientos para el diálogo corporal y el crecimiento perceptivo, expresivo y motriz.

Analizando los potenciales contenidos hacia el conocimiento del entorno a través de la caminata, el bloque «el cuerpo y la propia imagen» parte de los contenidos curriculares de expresión corporal, del área de educación física, facilita la utilización de las sensaciones y percepciones sensoriales. Mediante el bloque «juego y movimiento», se abren alternativas para la exploración y valoración de las posibilidades corporales, y las nociones básicas de orientación corporal. A través de "la actividad y la vida cotidiana», se pueden promover la actitud positiva con los demás, y gracias al bloque «el cuidado personal 
y la salud» se podrán practicar la aceptación de normas durante los desplazamientos y colaboración en el mantenimiento de ambientes y materiales limpios y ordenados.

Desde el área de "conocimiento del entorno», se intenta favorecer el descubrimiento del entorno infantil, incluyendo lo que afecta particularmente a cada uno y a los diferentes colectivos de pertenencia, interpretando su dimensión física, natural, social y cultural. El niño podrá conocer e interactuar los materiales de su entorno, manipulando y valorando aquellos no que constituyan un peligro. Esta es una de las ideas principales del aprendizaje, tal como realizan en las escuelas infantiles danesas situadas en el bosque. El niño irá descubriendo su pertenencia al medio social a través de experiencias escolares y culturales, que le ayudarán a alcanzar actitudes de aprecio hacia las mismas.

Al hablar de los contenidos de esta área, el bloque «medio físico: elementos, relaciones y medida» permitirá explorar los objetos y materias del medio, así como realización de desplazamientos orientados y posicionamiento de sí mismo y de los objetos en el espacio. Por su parte, el bloque «acercamiento a la naturaleza» plantea la importancia para su salud y bienestar de disfrutar del contacto con la naturaleza y los fenómenos del medio natural; y, a través del bloque «cultura y vida en sociedad», se observarán los modos de vida, ocupaciones e identidades familiares y culturales.

También el Ministerio de Educación, en el Real Decreto 126/2014 de Escolarización Primaria, considera la adquisición de competencias como un proceso continuo, respaldado en la participación en prácticas sociales dentro del contexto educativo formal, no formal e informal, generando un saber hacer que se pueda transferir a la variedad de contextos académicos y sociales. Con esta intención, se incorpora, en el currículum de Educación Primaria, elementos para formar parte del comportamiento relacionados con el medio ambiente y desarrollo sostenible, seguridad vial y la actividad física. Entre otras acciones, se contempla la práctica diaria de deporte y ejercicio físico por parte del alumnado durante la jornada escolar, cuyo diseño y supervisión serán asumidos por el profesorado.

\section{CoNOCIMIENTO DEL ENTORNO MEDIANTE HÁBitos ACTIVOS Y SALUDABLES DESDE LA EDUCACIÓN INFANTIL: LA CAMINATA RUMBO A LA ESCUELA COMO EJEMPLO}

Persiguiendo el conocimiento y disfrute del entorno, a través de la actividad física entre el alumnado y la comunidad educativa, con el mayor beneficio personal y social, se presentan diferentes propuestas desde la educación infantil 
(Thompson, Rivara y Thompson, 2005; Blanco y Ruiz, 2011; Chillón, Evenson, Vaughn y Ward, 2011; Dunlap, 2013; Organización Mundial de la Salud (WHO), 2010; St. Leger, Young y Perry, 2010), pues el entorno es un potente atractivo para fomentar la curiosidad y hábito exploratorio en el niño.

La tendencia de cualquier persona es moverse por el entorno realizando el itinerario más corto y natural, no siempre el señalizado. Las características de la vía podrían impedir un trazado natural, por lo que la propuesta de la persona es siempre la más segura y adaptada a sus condiciones. Aquellas experiencias en las que tengan que ubicarse en las instalaciones (patio escolar, parque público, pabellones de una granja-escuela, etc.) le ayudarán a ir seleccionando las mejores alternativas de caminos a realizar. De la misma forma, será igualmente productivo utilizar los espacios del recinto escolar y exteriores para las clases de educación física y juegos durante los descansos o en horario extraescolar. En cualquier caso, los profesores y padres deberán proporcionar el tiempo diario necesario para que los nińos tengan acceso a áreas de juego que apoyen el desarrollo físico de forma segura, dentro del recinto escolar o fuera, por lo que cobra mucha importancia el camino y la interacción comunitaria hacia ellos.

Un enfoque integrador sobre el conocimiento del entorno y la promoción de hábitos de vida activa lo constituye la caminata rumbo a la escuela, pues cuenta con una gran potencialidad social y educativa (Ayuntamiento de Santiago de Compostela, 2015; Berenguer, Burgos, Ortuño y Prieto, 2014; Logan, Corben, Oxley, Liu y Corben, 2013), pues da prioridad, en las calles, a los más jóvenes, educando en valores, promoviendo la convivencia vecinal y potenciando la interdisciplinaridad educativa con acciones sobre el entorno. Para ello, necesita la implicación del centro escolar, del profesorado y de las familias, pero también es importante poner en marcha grupos de trabajo que evalúen los trayectos, en los que participen los niños, para identificar los problemas existentes entre el espacio vivido y el real (Ascher, 2005; Overgaard, 2002). Para contribuir a crear un ambiente propicio dentro de una comunidad educativa y establecer un camino escolar que tenga continuidad en el tiempo, tiene que existir previamente una conciencia positiva de sus beneficios, para el niño, pero también para el barrio en el que se localice. Por eso, actuar desde la promoción de los hábitos de vida saludable y actividad física es acertado, pues es un recurso amplio, que llega a toda la población y lugares, y que puede dirigirse para mejorar el entorno, desde dentro o fuera de la escuela.

Para compartir el trayecto diario de la caminata rumbo a la escuela, entre las familias y los niños, es una buena actividad para evitar el sedentarismo, aumentar el rendimiento y la atención del alumnado desde las primeras horas 
lectivas, concienciando así hacia formas limpias de desplazamiento. También, desde el centro escolar, se pueden programar salidas en las que se realicen recorrido en los que los alumnos colaboren entre sí y con otras personas, fomentando las capacidades de observación y memorización del entorno, básicas para mejorar la autonomía en el desplazamiento de los niños, y se ponga de relevancia los valores que se desarrollan.

Tanto en actividades en la escuela como fuera del recinto escolar, es labor docente mejorar las habilidades perceptivas y de reacción motriz del niño, pues su baja estatura, imprevisibilidad de reacciones, reducido campo visual y nivel de maduración influyen en la capacidad de interpretación del espacio público y vehículos en circulación, y decisivamente en el riesgo de accidente, ya que pueden no ser vistos por los conductores. También será importante desarrollar hábitos de autoprotección en los niños en sus desplazamientos en bicicleta, como puede ser el uso del casco para reducir el riesgo de lesiones en la cabeza y cerebrales graves. Así, en cualquier tipo de práctica de iniciación en el centro escolar o en casa, será un elemento indispensable con el que el niño se identificará desde pequeño.

En cualquier momento, es interesante conocer a fondo el entorno próximo del centro escolar y aprovechar para explorar, con los niños, nuevas formas de interactuar con los espacios públicos, tanto los que estén en uso como aquellos que hayan sido abandonados. Una pequeña salida de forma regular ayudará a los niños a familiarizarse con los elementos físicos, naturales y sociales de los espacios cercanos, y observar que se realizan múltiples actividades diferentes a lo largo del día. Estas salidas a los alrededores pueden estar acompañadas de las familias, propiciando su participación en las actividades escolares de conocimiento e interacción con el entorno, mientras también realizan una ligera actividad física y comprueban la calidad de los caminos y las habilidades que vayan adquiriendo sus hijos. Es fundamental la concienciación de los padres para que sus hijos vayan caminando al colegio.

\section{EXPERIENCIAS DE IMPLANTACIÓN DE LA CAMINATA RUMBO A LA ESCUELA}

Ejemplos de camino escolar existen a lo largo y ancho de todo el planeta, cada vez en más lugares y con modelos de mayor complejidad. Históricamente, el camino escolar surgió en la ciudad danesa de Odense en los años 70, por la preocupación de la seguridad por los nińos que acudían al colegio andando o en bicicleta. No obstante, hasta los ańos 90, este tipo de proyectos no llegarían al centro de Europa, Australia, o Norteamérica. Su aparición coincidiría con la inquietud sobre el aumento de la circulación, la mayor conciencia ambiental, 
y los problemas de salud que comenzaba a generar la reclusión infantil. Los primeros proyectos internacionales se apoyaron en la seguridad y la prevención de los problemas de salud (Román y Salís, 2010), pero desde hace décadas, son muchos los países que han actuado en sus barrios y en las zonas próximas a los colegios.

En 1991, surgió el proyecto "Ciudad de los niños», con el objetivo de que estos saliesen de sus casas sin acompañante (Tonucci, 2014). La ciudad italiana de Roma fue la referencia entre 2001-2008 para las grandes urbes europeas, aunque la ciudad danesa de Odense fue la pionera en establecer una ruta segura al colegio, según la National Center to Safe Routes to School (NCSRS) (2016), desarrollando 108 actuaciones entre 1986 y 1999, además de proporcionar información muy valiosa sobre diseńo de carreteras o accidentes, para su mejora crítica y de otras localidades (NCSRS, 2016a). Con una fuerte implantación del proyecto SRTS, desde 1997, el Reino Unido coordina el establecimiento del School Travel Plan (STP) y el Sustainable Travel Accreditation and Recognition for Schools (STARS) (2016b) que, además de Londres, implicó a otras nueve ciudades europeas, llegando a 51.000 estudiantes y ahorrando 894 toneladas de $\mathrm{CO}_{2}$ atmosférico, por el aumento de formas de desplazamientos activos (STARS, 2016c). El proyecto Safety Road To School (SRTS), llegaría a Canadá en 1996 como un plan modelo de rutas escolares a pie, creciendo hasta convertirse en un importante movimiento nacional de movilidad escolar (Green Communities, 2016).

Más del 90\% de muertes del mundo, en las carreteras, ocurren en países con ingresos medios y bajos, que tienen menos de la mitad de vehículos del mundo. Aproximadamente, el $62 \%$ de las muertes por tráfico ocurren en 10 países, que en orden de magnitud son India, China, los Estados Unidos, la Federación de Rusia, Brasil, Irán, México, Indonesia, Sur África y Egipto, y representan 56\% de la población mundial.

En la región de América, peatones, motociclistas y ciclistas representan el $23 \%, 15 \%$ y $3 \%$ de muertes por tráfico rodado, respectivamente. La subregión de Mesoamérica tiene la mayor proporción de muertes de peatones, 30.8\%. Han sido numerosas las actuaciones para intentar mejorar la mortalidad y el tráfico tanto en las grandes ciudades como en las más pequeñas. En Bogotá, por ejemplo, la capital de Colombia, se construyeron durante la década pasada cientos de kilómetros de rutas ciclistas y para peatones, excluyendo a los automóviles del centro de la ciudad en horas punta en la mañana y la tarde, y desarrollando una mayor capacidad en el sistema de autobuses (Pan American Health Organization, 2013). 


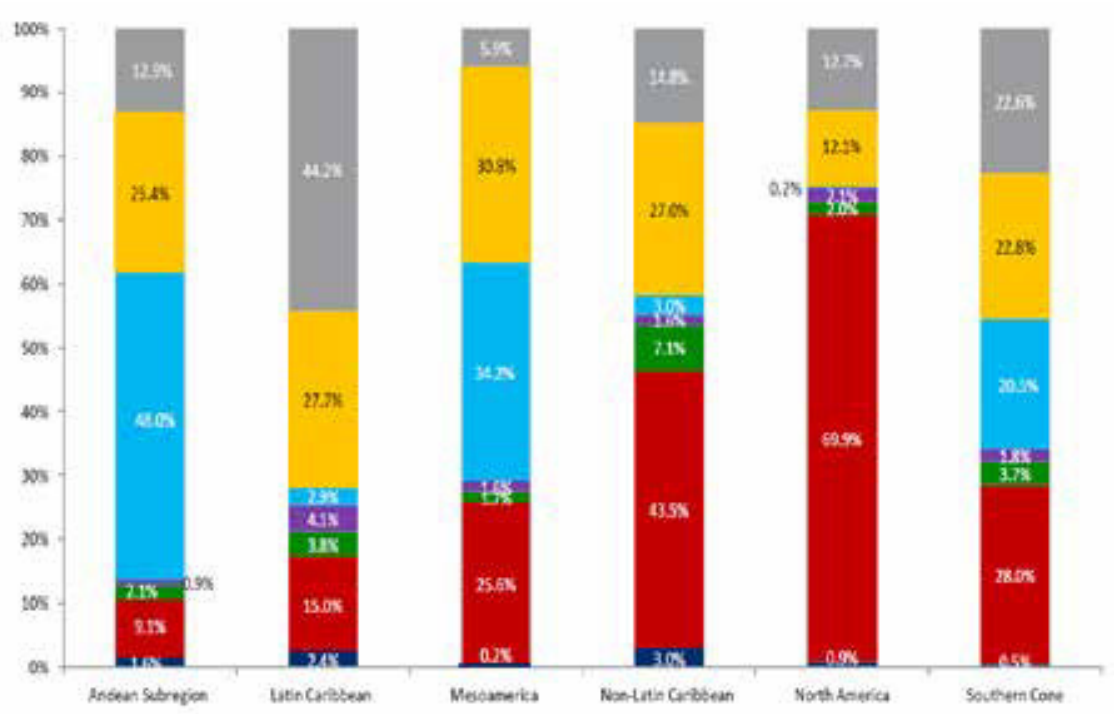

Ocupantes de bus - Ocupantes de camiones - Ocupantes de coches Ciclistas - Peatones - Motociclistas

Figura 1. Mortalidad en América según tipo de transporte. Fuente: Global Status Report on Road Safety. PAHO, 2013)

Desde la década de 1970, las «rutas seguras a la escuela» se han convertido en una parte reconocida de la cartera recomendada de políticas de transporte sostenible y seguridad vial. Los países de altos ingresos tienen programas en curso o algunas prácticas implementadas que han demostrado el valor y la eficacia de un enfoque de seguridad vial en el viaje a colegio.

El Proyecto SRTS desembarcaría en Estados Unidos en 1997, consiguiendo proyectos fuertemente financiados en California y Massachusetts, cuyo éxito ayudó al desarrollo de programa nacional (NCSRS, 2016b). En este país, por ejemplo, financiado por el Departamento de Transporte (DOT), ha invertido en aceras, cruces y diseño mejorado más de $\$ 1,2$ mil millones desde 2005 (Unicef, 2015).

El Grupo de Trabajo de la Casa Blanca sobre la Obesidad Infantil, establecido por el presidente Barack Obama, incluyó una serie de recomendaciones fuertes para continuar y aumentar la inversión en rutas seguras para escuelas, aliadas a un objetivo recomendado de aumentar en un $50 \%$ el porcentaje de niños entre 5 y 18 ańos tomando caminatas seguras y en bicicleta hacia y desde la escuela (White House Taskforce on Childhood Obesity, 2010). 
En todo el mundo, se encontró que más del $80 \%$ de los padres en Brasil, India y Sudáfrica estaban preocupados por la seguridad de su hijo caminando a la escuela (Safe Kids Worldwide, 2014). Un sistema vial seguro es relevante para el desarrollo de los países sudamericanos, tanto en los países de altos como de bajos ingresos. El primer paso es, quizás, el más difícil: reconocer el problema y compromiso político seguro.

En un objetivo de política adoptado por el presidente Kirchner (International Transport Forum Annual Report, 2014), Argentina, está tomando medidas en su seguridad vial y reducir el número de muertes. También la presidenta Dilma Rousseff de Brasil ha demostrado liderazgo, instando a la atención a la seguridad vial en su discurso de 2012 a la Asamblea General de las Naciones Unidas y ofreciendo albergar una conferencia al más alto nivel mundial. También, así como otros países en América Latina, como Argentina, Chile y Jamaica, Brasil está haciendo muchos esfuerzos considerables para reducir las víctimas del tránsito (International Transport Forum Annual Report, 2014).

También, en Uruguay, se ha avanzado en seguridad vial con la aprobación de una nueva legislación, tanto en los automóviles, autobuses, como ciclistas, motociclistas y peatones. En abril de 2010, un vehículo de transporte escolar se estrelló a solo cuatro cuadras de su destino, el Colegio Pallotti, lo que ocasionó la muerte de Julieta Estefan de 11 años. Otros dos niños resultaron gravemente heridos. El autobús tenía cinturones de seguridad en el frente para el conductor y los maestros, pero no para los niños. En mayo de 2013, tuvo lugar un choque casi idéntico, cuando un autobús escolar colisionó con un automóvil en Montevideo, en el área del centro, y volcó. Todos los niños sufrieron no más que lesiones menores, ya que eran usando cinturones de seguridad debidamente abrochados (Unicef, 2015)

En 2001, se iniciaba, en Nueva Zelanda, el Walking School Bus, un proyecto de pedibús a la escuela (New Zealand Transport Agency, 2009), que se ampliaría con el School Travel Plan, alcanzando en 2012 las 319 escuelas.

En España, existen numerosas iniciativas en todas las principales ciudades y localidades de diferentes tamaños. Los inicios fueron en Barcelona (Cataluña) en el año 1999, dando paso en 2004 al proyecto Camino Escolar Seguro, que alcanzaría al 36\% de la población escolar en 2012 (Laria del Vals et al., 2014). En 2003, aparecerían iniciativas en las regiones de Galicia, País Vasco y Cataluña, y, posteriormente, en Madrid, Andalucía, y el resto del país.

En la capital, la propuesta Madrid a pie, camino seguro al cole, implicó a 22 centros educativos de Educación Primaria, aumentando un 9,9\% el transporte a pie. La ciudad de San Sebastián (País Vasco) implementó SWITCH, un programa europeo que generó diferentes recursos prácticos sobre movi- 
lidad escolar e infantil (Consorcio SWITCH, 2015). Actualmente, la Agencia Energética de la Ribera de Valencia (AER) (2016) está inmersa en el proyecto de promoción de los desplazamientos a pie de los escolares: «Let's move!», con la participación de 15 centros escolares locales, y otros 11 de Francia, Polonia y Croacia. Por su parte, en Pontevedra (Galicia), su plan de ciudad ha obtenido numerosos premios internacionales, contribuyendo desde 2011 a desarrollar un programa de caminos escolares de referencia para otras ciudades (Ayuntamiento de Pontevedra, 2016).

Tal y como apunta el Departamento de Medio Ambiente - Comisiones Obreras de Aragón (DMAA-CCOO), el protagonista del camino escolar es el alumno, que aprenderá a desplazarse con seguridad y por sus propios medios, y a reflexionar en actividades de aula sobre movilidad y mejora ambiental, promocionadas por directivos y profesores (DMAA-CCOO, 2012). Por otra parte, es fundamental el apoyo de las familias y asociaciones de madres y padres, pues ellos decidirán sobre los hábitos de movilidad de sus hijos (Román y Salís, 2010). También es importante, para la Dirección General de Tráfico (DGT) (2016), el vecindario y el tejido asociativo, de gran valor para establecer una relación de confianza con los nińos y adultos. Y, por supuesto, incluyendo a los cuerpos y fuerzas de seguridad del Estado, el papel de la administración pública, que cuenta con la capacidad de invertir e intervenir con ayuda diversa en los trámites e implantación, además de la capacidad de coordinarse con otros servicios y realizar campañas de concienciación (Chillón, Evenson, Vaughn y Ward, 2011).

Entre las formas de camino escolar más representativas, nos encontramos con el camino libre o itinerario por vías señalizadas y seguras para que los nińos puedan acudir solos al colegio, tanto en bicicleta como andando. El pedibús, o itinerario, con paradas para recoger a los alumnos e ir al colegio andando, solos o acompañados de adultos voluntarios, generalmente uno en cabeza y otro cerrando el grupo; y el bicibús o grupo al colegio en bicicleta, solos o acompańados por adultos, utilizando vías de circulación más restringidas, para no entorpecer el espacio de peatones.

Entre los ańos 1999 a 2016, un total de 739 centros escolares de Educación Primaria e Infantil entre Espańa y Andorra han generado un proyecto de camino escolar, como se puede apreciar en la figura 1, de los que el $6 \%$ continúan en fase de diagnóstico, el $89 \%$ se mantienen activos y el $11 \%$ ya han finalizado. 


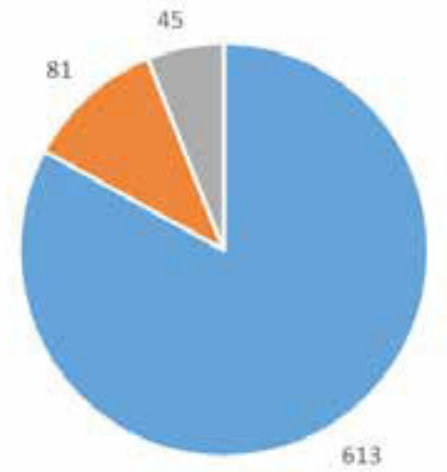

- Activos a Finalizados an diagnóstico

Figura 2. Situación de los Proyectos de Camino Escolar en España y Andorra 1999-2016. Fuente: Silva y Daquinta, 2016

En la figura 2, se aprecia que en todas las regiones españolas existe al menos un proyecto de camino escolar de referencia, aunque, entre Andalucía y Cataluña, se concentra casi el $50 \%$ de todas las iniciativas locales actuales y solo en la provincia de Barcelona (Cataluña) se localizan el 95\% de sus proyectos regionales, algo más del $20 \%$ del total nacional.

En cuanto a la tipología del promotor del proyecto local de camino escolar, en el $85 \%$ de los casos el ayuntamiento o sus servicios afines (policía local), es el auténtico impulsor de este tipo de iniciativas, apoyados en su capacidad de promoción social, como en las posibilidades de captación y coordinación de recursos y personas que tienen (Figura 3).

Generalmente, son los departamentos municipales de urbanismo o tráfico los líderes, pero, inmediatamente, entran a colaborar internamente con otros departamentos, como educación, medio ambiente, asuntos sociales, salud o bienestar social, pues todos los factores están interrelacionados y es inevitable optimizar recursos y crear sinergias que puedan acelerar el proyecto. También aparecen, entre los promotores principales, aunque de forma menos relevante (12\%), el propio centro escolar, las diputaciones provinciales, o las asociaciones de madres y padres de alumnos (AMPAS). 


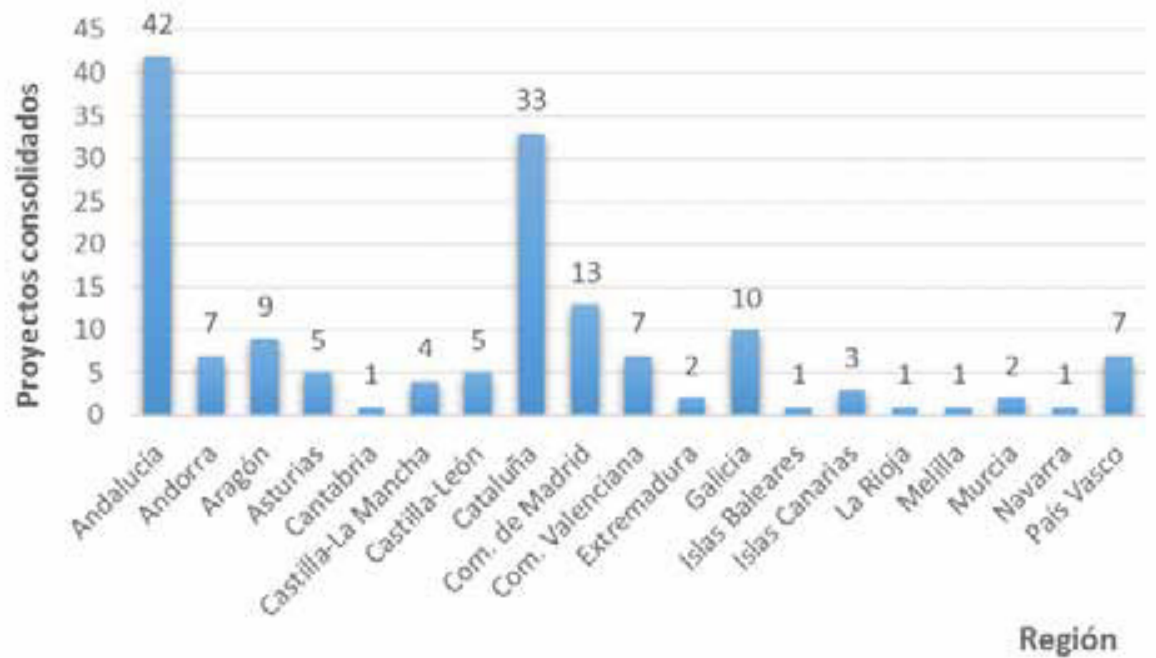

Figura 3. Distribución regional de los Proyectos de Camino Escolar en España y Andorra 1999-2016. Fuente: Silva y Daquinta, 2016

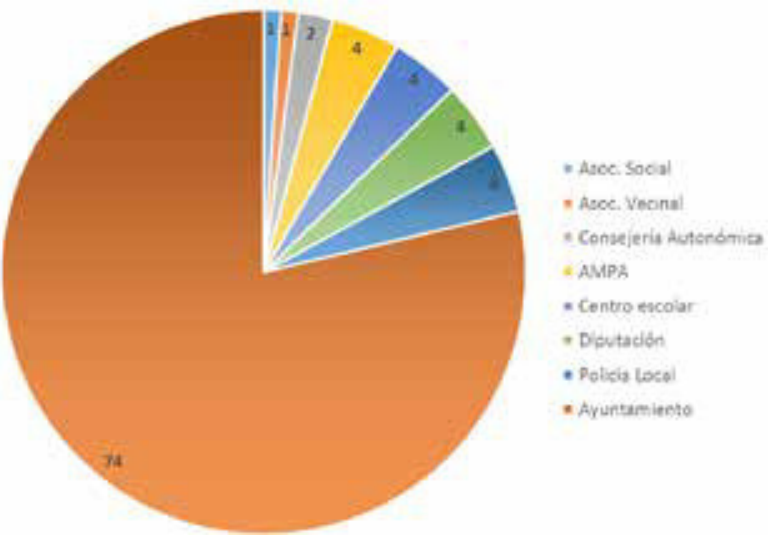

Figura 4. Promotores de los Proyectos de Camino Escolar en España 19992016, según porcentaje de representatividad. Fuente: Silva y Daquinta, 2016

De modo igualmente relevante, para comenzar y mantener en el tiempo cualquier proyecto de camino escolar son fundamentales los colaboradores directos y estratégicos, entre los que destaca el centro escolar (88\%), por la implicación en la finalidad del proyecto (Figura 4). En aquellas propuestas que 
no lidera el centro escolar, también es importante la colaboración del ayuntamiento (7\%), o con las AMPAS y otros colectivos (5\%).

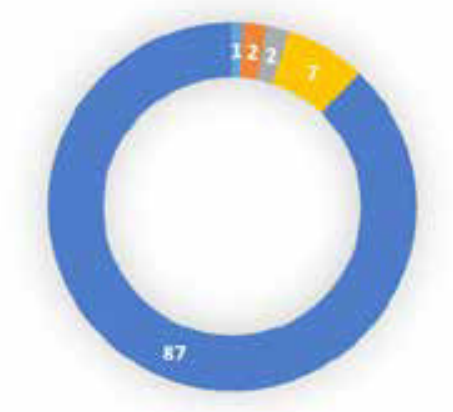

wConsutoria =AMPA =FEDEF-UE =Ayuntamiento wCentroescolai

Figura 5. Colaboradores directos de los Proyectos de Camino Escolar en España 1999-2016 según porcentaje de representatividad. Fuente: Silva y Daquinta, 2016

\section{ASPECTOS PARA CONSIDERAR EN EL FOMENTO DE ENTORNOS ESCOLARES ACTIVOS}

Tal y como se señala en la tabla 1, es adecuado no focalizar el fomento de entornos escolares activos parcialmente en el alumnado o profesorado, sino siempre favorecer el bienestar de ambos, siendo los estudiantes lo primero, y anteponiendo, en ocasiones, la seguridad y salud del profesorado.

En cualquier caso, la labor docente es prioritaria para avanzar en cualquier tipo de proyecto educativo, por eso es necesario capacitar a los profesores en la implementación de los entornos escolares activos, que contemplen el fomento y control del conocimiento del ambiente a través de la mayor movilidad y actividad física para la salud. En este sentido, los maestros necesitarán recibir información y formación para incorporar temas efectivos relacionados en su área temática, pues todos ellos deben servir como modelos responsables para los estudiantes. 
Programar actividades durante períodos de menor contaminación ambiental

Evitar que la contaminación entre directamente al edificio de la escuela y mejorar la protección vegetal

Reforzar las actividades que favorezcan los hábitos activos posteriormente en la escuela

Favorecer el bienestar de toda la comunidad escolar, principalmente alumnos y profesores

Capacitación del profesorado en temáticas específicas sobre el entorno escolar activo para sus respectivas áreas o materias

Implicar a las familias en el diseño y cuidado de espacios interiores y exteriores para los hábitos activos

Mantener una buena relación y contacto periódico con entidades del entorno próximo

Integrar las propuestas de entorno escolar activo en el Proyecto Educativo de Centro, realizar nombramiento de cargos específicos y la creación de comisiones específicas

Revisión de señalización, plan de emergencia, limpieza y organización de espacios

Incentivar la adaptación de los espacios para mejorar sus condiciones de práctica activa, haciendo partícipe directamente al alumnado y personal del centro

Mejorar el drenaje y cuidado de los patios y espacios exteriores

Tabla 1. Aspectos para considerar en el fomento de entornos escolares activos. Fuente: Modzeleski et al., 2012; Burgos, 2010; Longás, 2012; González y Casado, 2005

Revisar periódicamente la señalización del centro y los aspectos relacionados con el plan de emergencia y establecer unas normas básicas para el orden y limpieza de los espacios escolares y exteriores, contando con el apoyo del personal, alumnado y profesorado. Asimismo, requerirán incentivar el cuidado y los cambios de los espacios escolares a través de actuaciones con el alumnado, que mejoren las condiciones de prácticas activas y actualizadas a 
sus necesidades. De igual forma, es importante para ellos implicar a las familias en el diseńo y cuidado de espacios e itinerarios para la práctica física y hábitos activos dentro del recinto escolar, que puedan ser considerados para cualquier otra propuesta fuera del colegio.

Entre las medidas de calidad y seguridad de la práctica al aire libre, destaca la necesidad de mejorar el drenaje y cuidado de los patios y espacios exteriores, y programar actividades durante períodos del día en que la contaminación atmosférica y la exposición al sol son más bajas. Algunas escuelas tendrán que preocuparse por la calidad del aire exterior, evitando el ejercicio físico intensivo al aire libre durante períodos de alta contaminación del aire. La probabilidad de que los nińos con asma tengan un ataque se estima en un $40 \%$ mayor en los días de alta contaminación al aire libre, teniendo en cuenta también que los niños que juegan a deportes activos son más propensos a desarrollar la enfermedad en las ciudades contaminadas (Organización de Consumidores y Usuarios, 2012).

Evitar que la contaminación entre directamente al edificio de la escuela, sobre todo durante los momentos de máxima afluencia, se aconseja mejorar la protección vegetal del recinto escolar, que además ayudará a reducir la sobreexposición a los rayos ultravioleta del sol.

Siempre es acertado mantener una buena relación y contacto periódico con entidades del entorno próximo, haciéndose responsables recíprocos de los proyectos, planes y acciones desde las escuelas, que contribuyen a la creación y mantenimiento de entornos escolares seguros y saludables, y la organización de actividades físicas escolares y sociales. En este sentido, se requiere aprovechar las sinergias para que los nińos pequeńos participen en excursiones por el entorno natural con otros mayores o adultos, y que estos puedan ayudarles en aquellos que necesiten. A posteriori, es importante recordar y reforzar en la escuela las experiencias vividas en la realización de estos itinerarios.

Para lograr la mayor eficacia y garantías educativas, tanto los proyectos de entorno escolar activo como los relacionados con el camino escolar, debieran integrarse en el Proyecto Educativo de Centro, realizando el nombramiento de cargos específicos y la creación de comisiones que promuevan los ambientes seguros y saludables, y organizando un grupo de trabajo en el Consejo Escolar y un Comité de Salud y Movilidad Escolar, con la misión de asegurar que las instalaciones, los itinerarios y los parques son seguros y saludables para los niños. 


\section{REFERENCIAS BIBLIOGRÁFICAS}

Agencia Energética de la Ribera (2016). Project Let's Move. Recuperado de https://projectletsmove.wordpress.com/

Alonso, F., Esteban, C., Calatayud, C. y Alamar, B. (2009). Los niños, las ciudadesy la seguridad vial: una visión a partir de la investigación. Valencia: Attitudes.

Ascher, F. (2005). Los nuevos principios del urbanismo. Madrid, España: Alianza Editorial, S.A.

Asociación Española de Pediatría de Atención Primaria (2014). Accidentes en población infantil española. Fundación MAPFRE. Recuperado de https:// www.fundacionmapfre.org/fundacion/es_es/seguridadvial/investigacion/ accidentes-poblacion-infantil-espanola.jsp

Ayuntamiento de Pontevedra (2016). Camiño escolar. Pontevedra. Recuperado de http://www.pontevedra.eu/movete/camino-escolar/presentacion

Ayuntamiento de Santiago de Compostela (2015). Colecamiños! Camino escolar seguro. Santiago de Compostela. Recuperado de http://policialocal.santiagodecompostela.gal/media/documentos/folletowebColeca minos.pdf

Berenguer, P., Burgos, J.R., Ortuńo, J. y Prieto, A. (2014). Proyecto caminos escolares seguros. Alicante. Recuperado de: http://age-tig.es/2014_Alicante/ ponencia3/113.pdf 22

Blanco, A. y Ruiz, F. (2011). La movilidad segura de los colectivos más vulnerables. La protección de peatones y ciclistas en el ámbito urbano. Observatorio Nacional de Seguridad Vial. Dirección General de Tráfico. Recuperado de http://www.dgt.es/Galerias/la-dgt/centro-de documentación/publicaciones/2011/doc./la-movilidad-segura-de-los colectivos-mas-vulnerables. pdf

Burgos, A. (2010). ¿Cómo integrar la seguridad y salud en la educación? Elementos clave para enseñar prevención en los centros escolares. Profesorado. Revista de currículum y formación del profesorado, 14(2), 273-301.

Chillón, P., Evenson, K., Vaughn, A. y Ward, D. (2011). A systematic review of interventions for promoting active transportation to school. International Journal of Behavioral Nutrition and Physical Activity, 8(1), 10. https://doi. org/10.1186/1479-5868-8-10

Chillón, P., Ortega, B., Ruiz, J., Evenson, K., Labayen, I., Martínez-Vizcaíno, V., Hurtig-Wennlöf, A., Veidebaum, T. y Sjöström, M. (2012). Bicycling to school is associated with improvements in physical fitness over a 6-year follow-up period in Swedish children. Preventive Medicine, 55(2), 108112. https://doi.org/10.1016/j.ypmed.2012.05.019 
Confederación Española de Asociaciones de Padres de Alumnos (2012). Cómo construir una escuela segura y saludable. Guí para padres. Recuperado de http://goo.gl/t27P6i.

Consorcio SWITCH (2015). Guia para campañas SWITCH: Consejos prácticos para campañas que promueven la sustitución de viajes en coche por modos activos de desplazamiento. Colonia. Recuperado de http://www.switchtravel.eu

Danish Cancer Society (2012). Healthy and bicycle-friendly school road. Recuperado de https://www.cancer.dk/dyn/resources/File/file/0/840/1385267414/ healthyandbicyclefr iendlyschoolroads.pdf

Danish National Board of Health (2006). Physical activity and evidence - Lifestyle diseases, endemic diseases and risk factors. En Danish Cancer Society (2012). Healthy and bicycle-friendly school road. Recuperado de https://www. cancer.dk/dyn/resources/File/file/0/840/1385267414/healthyandbicyclefr iendlyschoolroads.pdf. 23

Davison, K.K., Werder, J.L. y Lawson, C.T. (2008). Children's active commuting to school: current knowledge and future directions. Preventing Chronic Disease, 5(3), A100.

Departamento de Medio Ambiente - Comisiones Obreras Aragón (2012). Caminos escolares. Zaragoza. Recuperado de https://www.zaragoza.es/contenidos /viapublica/caminos/Def_CCOO_Camino\%20Escolar.pdf

Devine, J. y Cohen, J. (2007). Making your school safe: strategies to protect children and promote learning. Nueva York: Teachers College Press.

Dirección General de Tráfico (2016). Camino escolar, paso a paso. Madrid. Recuperado de http://www.dgt.es/PEVI/contenidos/Externos/recursos/ infancia/camino_escolar/ca mino_escolar_Paso_a_Paso.pdf

Dunlap, S. (2013). The comprehensive handbook of school safety. Boca Raton, FL: Taylor \& Francis Group. https://doi.org/10.1201/b12700

European Commission (2014). Special Eurobarometer 412. Sport and Physical Activity. Recuperado de http://ec.europa.eu/health/nutrition_physical_ activity/docs/ebs_412_en.pdf

European Heart Network (2001). Children and Young People - the Importance of Physical Activity. Recuperado de http://ec.europ.eu/health/ph_projects/ 2000/promotion/fp_promotion_2000_frep_11_en.pdf.

Gavidia, V. (2001). La transversalidad y la escuela promotora de salud. Revista Española de Salud Pública, 75(6), 505-516. https://doi.org/10.1590/ S1135-57272001000600003

González, J. y Casado, S. (2005). Formación de los trabajadores en materia de prevención de riesgos laborales. Prevention World Magazine: prevención de riesgos, seguridad y salud laboral, 7, 52-55. 
Green Communities (2016). Canada walks. Recuperado de http://greencommunitiescanada.org/programs/canada-walks/

Hamer, M. y Chida, Y. (2008). Active commuting and cardiovascular risk: a metaanalytic review. Preventive Medicine, 46, 9-13. https://doi.org/10.1016/j. ypmed.2007.03.006

Hayes, D. (2003). Planning, teaching and class management in primary schools. Oxon (GB): Routledge. 24

International Transport Forum Annual Report (2014). International Transport Forum Annual Report. Recuperado de http://www.oecd-ilibrary.org/transport/road-safety-annual-report-2014_irtad-2014 en; jsessionid $=44$ iavc03pq5ev.x-oecd-live-03

Laria del Vals, J., Monclús González, J., Ortega Pérez, J. y Equipo del Área de Seguridad Vial de la Fundación Mapfre (2014). Manual de Inspecciones de Seguridad Vial en entornos seguros. Recuperado de https://www.fundacionmapfre.org/fundacion/es_es/images/Manual-auditorias-entornosescolares_tcm 164-58428.pdf

Logan, D.B., Corben, B.F., Oxley, J., Liu, S. \& Corben, K. (2013). A model for star rating school walking routes. Australia. Recuperado de https://trid.trb. org/view.aspx?id=1279309

Longás, J. (2012). Sobre el benestar a l'escola i en el professorat. Estudis i aproximació conceptual. En J. Longás y M. Martínez (2011), El benestar als centres $i$ en el professorat. L'estat de l'educació a Catalunya. Anuari 2011 (pp. 21-102). Barcelona: Fundació Jaume Bofill.

Lubans, D. R., Boreham, C. A., Kelly, P. y Foster, C. E. (2011). The relationship between active travel to school and healthrelated fitness in children and adolescents: a systematic review. International Journal of Behavioral Nutrition and Physical Activity, 8, 5. https://doi.org/10.1186/1479-5868-8-5.

MacKay, M. \& Vincenten, J. (2012). Child Safety Report Card 2012: Europe Summary for 31 Countries. Birmingham: European Child Safety Alliance, Eurosafe.

McMillan, T. E. (2007). The relative influence of urban form on a child's travel mode to school. Transportation Research: Part A: Policy and Practice, 41(1), 69-79. https://doi.org/10.1016/j.tra.2006.05.011

Menéndez, C. y Moreno, F. (2006). Ergonomía para docentes. Análisis del ambiente de trabajo y prevención de riesgos. Barcelona: Graó.

Meresman, S. (2005). Escuelas promotoras de salud. En Borrell, R. (2005) Portafolio educativo. Municipios saludables (pp. 290-314). Buenos Aires: OPS / Ministerio de Salud y Ambiente de la Nación.

Ministerio de Educación, Cultura y Deporte (2006). Real Decreto 1630/2006, de 29 de diciembre, por el que se establecen las enseñanzas minimas del segundo 
ciclo 25 de Educación infantil. Recuperado de https://www.boe.es/diario_ boe/txt.php?id=BOE-A-2007-185

Ministerio de Educación, Cultura y Deporte (2014). Real Decreto 126/2014, de 28 de febrero, por el que se establece el curriculo básico de la Educación Primaria. Recuperado de https://www.boe.es/diario_boe/txt. php?id=BOE-A-2014-2222.

Modzeleski, W., et al. (2012). An introduction to the safe schools / healthy students initiative. Evaluation and Program Planning, 35(2), pp. 269-272. Doi: 10.1016/j. evalprogplan.2011.11.001

Muennin, P., Epstein, M., Li, G. y DiMaggio, C. (2014): The Cost-Effectiveness of New York City's Safe Routes to School Program. American Journal of Public Health. Vol. 104, 7, 1294-1299. Recuperado de http://ajph.aphapublications.org/doi/abs/ 10.2105/AJPH.2014.301868

National Center to Safe Routes to School (2016a). Odense: Safe Routes To School. Recuperado de http://www.saferoutesinfo.org/program- tools/ odense-safe-routes-children

National Center to Safe Routes to School (2016b). History of Safe Routes to School Recuperado de http://guide.saferoutesinfo.org/introduction/history_of_ srts.cfm

National Center to Safe Routes to School (2016c). The Decline of walking and bicicycling. Recuperado de http://guide.saferoutesinfo.org/introduction/ the_decline_of_walking_and_bicycling. Cfm

New Zealand Transport Agency (2009). Walking school bus, coordinator guide. Wellington. Recuperado de https://education.nzta.govt.nz/_data/assets/ pdf_file/ 0015/5145/wsb-coordinators- guide.pdf

Organización de Consumidores y Usuarios (2012). Contaminación a la puerta del cole. El enemigo invisible. Revista OCU, 362, 12-16.

Overgaard, J. (2002). Web-based questionnaires in transport studies. Trafikforskningsgruppen. Aalborg University. En Danish Cancer Society (2012) Healthy and bicycle-friendly school road. Recuperado de https:// www.cancer.dk/dyn/resources/File/file/0/840/1385267414/healthyandbicyclefr iendlyschoolroads.pdf 26

Pan American Health Organization (2011). Pedestreian Safety. SustainableDevelopmentandEnvironmentalHealth (SDE). Recuperado de: https://www.paho.org/hq/index.php?option=com_docman\&task=doc_ view\&Itemid=2 70\&gid=20376\&lang=en

Peden, M. et al. (eds.) (2004). World report on road traffic injury prevention. Geneva: WHO. Recuperado de http://www.who.int/violence_injury_prevention/publications/road_traffic/world_report/en/index.html. 
Román, M. y Salís, I. (2010). Camino escolar. Pasos hacia la autonomía infantil. Madrid, Espańa: Ministerio de Fomento. Gobierno de España.

Sælensminde, K. (2004) Cost-benefit analyses of walking and cycling track networks taking into account insecurity, health effects and external costs of motorized traffic. Transportation Research Part A 38 (2004), 593-606. https://doi.org/10.1016/j.tra.2004.04.003

Silva, R., y Daquinta, J. (2016): Proyectos de caminos escolares en centros de educación primaria en España y Andorra. VI Congreso Mundial del Deporte Escolar, Educación y Psicomotricidad. 3-5 noviembre. A Coruña.

Sirard, J. R., Ainsworth, B. E., McIver, K. L. y Pate, R. R. (2005). Prevalence of active commuting at urban and suburban elementary schools in Columbia, SC. American Journal of Public Health, 95(2), 236-237. https:// doi.org/10.2105/AJPH.2003.034355

Spallek, M., Turner, C., Spinks, A., Bain, C. y McClure, R. (2006). Walking to school: Distribution by age, sex and socio-economic status. Health Promotion Journal of Australia: Official Journal of Australian Association of Health Promotion Professionals, 17(2), 134-138. https://doi.org/10.1071/ HE06134

Safe Kids Worldwide (2014). Global Road Safety for Children report. Recuperado de: http://www.safekids.org/research-report/safe-roads-safekids-globalroad-safety-children december-2014

St Leger, L., Young, I.; \& Perry, M. (2010). Promover la salud en la escuela: de la evidencia a la acción. Saint Denis Cedex (Francia): uipes. Recuperado de http://goo.gl/ccoZ2x. 27

Sustainable Travel Accreditation and Recognition for Shools - STARS (2016a). Promoting active travel in schools. Londres. Recuperado de https://stars.tfl. gov.uk/PublicPages/Home.aspx

Sustainable Travel Accreditation and Recognition for Schools - STARS (2016b): Informe final de proyecto. Recuperado de http://starseurope.org/assets/ STARS\%20Final_Report_ES.pdf

Sustainable Travel Accreditation and Recognition for Schools - STARS (2016c): Boletin informativo del proyecto, abril 2016. Recuperado de http://starseurope.org/assets/STARS\%20Newsletter\%20April\%202016.pdf

Thompson, D. C., Rivara, F. P. y Thompson, R. (2005): Helmets for preventing head and facial injuries in bicyclists. Cochrane Database of Systematic Reviews, (4): CD001855.

Tonucci, F. (2014). La ciudad de los niños: un modo nuevo de pensar la ciudad. Madrid: Fundación Germán Sánchez Ruipérez. 
Unicef (2015). Safe journeys to school are a child's right. Recuperado de: https:// www.fiafoundation.org/media/.../safe-to-learn-report.pdf

Villa, E. y Pérez, I. (2014). El desplazamiento activo al colegio. Una propuesta de intervención escolar. Tándem Didáctica de la Educación Física, 46, 24-32.

Villa-González, E., Rodríguez-López, C., Huertas Delgado, F. J., Tercedor, P., Ruiz, J. R. y Chillón, P. (2012). Factores personales y ambientales asociados con el desplazamiento activo al colegio de los escolares españoles. Revista De Psicología Del Deporte, 21(2), 343-349.

Warburton, D., Nicol, C., Bredin, S. (2006). Health benefits of phsysical activity: the evidence. CMAJ, 174 (6): 801-9. Recuperado de http://www.ncbi.nlm. nih.gov/pubmed/16534088

Westerterp, K.R. y Speakman, J.R. (2008). Physical activity energy expenditure has not declined since the 1980s and matches energy expenditures of wild mammals. International Journal of Obesity, 32(8), 1256-1263. https://doi. org/10.1038/ijo.2008.74

White House Taskforce on Childhood Obesity (2010), Report to the President: Solving the Problem of Childhood Obesity Within A Generation. Recuperado de: http://www.letsmove.gov/white-house-task-force-childhood-obesity-report-president 28.

World Health Organization (2008): WHO mortality database: tables. Geneva: World Health Organization. Recuperado de http://www.who.int/ healthinfo/mortables/en/index.html.

World Health Organization (2010). Global recommendation on Physical Activity for Health. Geneva. Recuperado de whqlibdoc.who.int/publications/2010/9789241599979_eng.pdf

World Health Organization-Regional Office for Europe (2014). Unlocking new opportunities: Jobs in Green and healthy transport. Recuperado de http:// www.euro.who.int/_data/assets/pdf_file/0003/247188/Unlocking-newopportunities-jobs-in-green-and-health-transport-Eng.pdf

Xunta de Galicia (2015). Movilidad sostenible y segura. Recuperado de http://www. edu.xunta.es/portal/es/node/17050 